\title{
Toward scalable information processing with ultracold polar molecules in an electric field: A numerical investigation
}

\author{
Laëtitia Bomble, ${ }^{1}$ Philippe Pellegrini, ${ }^{1}$ Pierre Ghesquière, ${ }^{1}$ and Michèle Desouter-Lecomte ${ }^{1,2}$ \\ ${ }^{1}$ Laboratoire de Chimie Physique, Université Paris-Sud, UMR 8000, Orsay F-91405, France \\ ${ }^{2}$ Département de Chimie, Université de Liège, Bât B6c Sart Tilman, B-4000 Liège Belgium
}

(Received 27 July 2010; published 22 December 2010)

\begin{abstract}
We numerically investigate the possibilities of driving quantum algorithms with laser pulses in a register of ultracold $\mathrm{NaCs}$ polar molecules in a static electric field. We focus on the possibilities of performing scalable logical operations by considering circuits that involve intermolecular gates (implemented on adjacent interacting molecules) to enable the transfer of information from one molecule to another during conditional laser-driven population inversions. We study the implementation of an arithmetic operation (the addition of 0 or 1 on a binary digit and a carry in) which requires population inversions only and the Deutsch-Jozsa algorithm which requires a control of the phases. Under typical experimental conditions, our simulations show that high-fidelity logical operations involving several qubits can be performed in a time scale of a few hundreds of microseconds, opening promising perspectives for the manipulation of a large number of qubits in these systems.
\end{abstract}

DOI: 10.1103/PhysRevA.82.062323 PACS number(s): 03.67.Lx, 03.67.Ac, 33.20.Bx, 37.10.Pq

\section{INTRODUCTION}

The past few years have witnessed remarkable experimental achievements in the realization of elementary quantum logical operations on physical systems [1-5]. Building a quantum processor that could handle a large number of quantum bits (qubits) represents the next milestone to reach toward the realization of a practical quantum computer, but no technology is currently available for processing about 100 qubits, which is the expected minimum number of qubits required to outperform powerful current classical computers. Due to their rich inner energy structure that can be used to encode information, molecules offer promising propects for scalable quantum-information processing and have attracted a lot of attention recently. Following the pioneering work of de VivieRiedle and co-workers [6,7], several groups have explored the possibility of encoding qubits in rovibrational states of a single diatomic molecule [8-18] or polyatomic molecule [19-29] or in two interacting diatomic molecules [30,31]. In these works, logic gates were driven by pulses designed by optimal control or genetic algorithms or by using stimulated Raman Adiabatic passage (STIRAP) techniques. From the viewpoint of scalability, it is challenging to increase the number of encoded qubits on a single molecule because the number of eigenstates individually addressable cannot grow exponentially. A more promising strategy would be to use a network of polar molecules holding a limited number of qubits [32]. Polar molecules are ideal systems for such a strategy because they can interact through the strong anisotropic long-range dipole-dipole interaction enabling couplings between qubits to create entanglement [32,33]. Furthermore, experimental progress obtained in the formation of stable heteronuclear alkali-metal molecules at ultracold temperatures $(\sim \mu \mathrm{K})$ opens up the possibility for an individual tight confinement and manipulation of the molecules by optical means, paving the way to the actual realization of a polar-molecule-based quantum register [32]. Different schemes have been proposed to realize universal quantum gates and manipulate qubits encoded on molecular levels. The realization of a conditional phase gate has been proposed by using either a switchable dipole-dipole interaction [34,35] or a sequence of laser pulses [36].

In this article, we present a numerical investigation of the possibilities of driving quantum algorithms with laser pulses on a register of polar molecules trapped in an optical lattice and experiencing a static electric field. A step toward scalability is addressed by considering schemes that involve intramolecular (implemented on a single molecule) and intermolecular (when interacting molecules are used) quantum gates. Encodings that use both the vibrational and rotational modes of the molecules are considered as well. We first implement the addition of 0 or 1 to a binary digit $b_{i}$ with a carry in $c_{i}$ to obtain the sum $s_{i}$ and the carry out $c_{i+1}$ [37]. This adder requires three qubits and never involves superposed states. In this sense the computation is weakly affected by dephasing. By omitting the phase constraint, the conditional population inversions can be realized by $\pi$ pulses. Implementing these arithmetic operations on molecular systems at this quasiclassical level should already be appealing for future applications. This logical scheme allows for the concatenation of several arithmetic operations by saving the sum $s_{i}$ in the vibration of one molecule for a latter reading and, most importantly, by transferring the carry out $c_{i+1}$ to a neighboring molecule enabling the next cycle of addition to be carried out without any intermediary readings. A careful treatment of the phases for quantum gates involving superposed states is realized in the context of the Deutsch-Jozsa algorithm [38] applied on a one-bit function. Similar to the intermolecular gates of the adder, the active qubit and the ancillary one are encoded in two neighboring molecules (see also [30] for a two-molecule implementation). Pulses are then designed by optimal control theory (OCT) with an additional constraint for the phase [6,7].

This article is organized as follows: in Sec. II, we give a detailed description of the polar-molecule-based quantum register [32] we consider as a support for realistic numerical simulations. We also explain the theoretical framework of our treatment. In Secs. III and IV, we present the conditional population inversion driven by $\pi$ pulses and the realization of a three-qubit 0 adder and 1 adder, respectively. Finally, in 

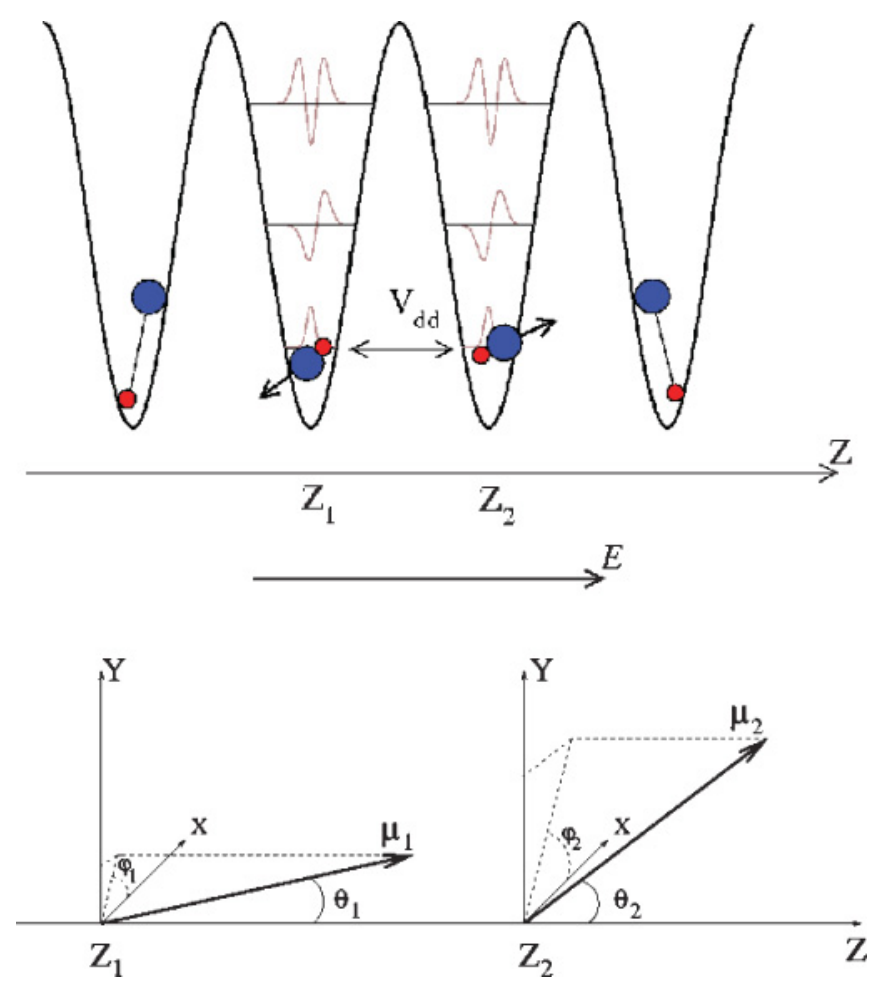

FIG. 1. (Color online) Top panel: Schematic of a polar-moleculebased quantum register [32]. Bottom panel: Permanent dipole moments $\mu_{i}, i=1$ and 2 , of the molecules. Their orientations are characterized by the angles $\theta_{i}$ and $\phi_{i}$. The electric field $E$ is parallel to the $Z$ axis connecting the center of mass of the molecules. In the simulation, the wave length of the lattice laser is $\lambda=600 \mathrm{~nm}$, so the intermolecular distance is $300 \mathrm{~nm}$, the dipole moment is $4.6 \mathrm{D}$, and the dipole-dipole interaction is about a few tens of $\mathrm{kHz}$.

Sec. V we present simulations of phase correct gates with application to the Deutsch-Jozsa algorithm.

\section{MODEL}

\section{A. Quantum register of polar molecules}

Figure 1 shows a schematic description of the polarmolecule-based quantum register. It consists of a string of polar ultracold heteronuclear $\mathrm{NaCs}$ dimers experiencing a static electric field [32]. In its ground $X^{1} \Sigma^{+}$electronic state, NaCs has a permanent dipole moment of $4.6 \mathrm{D}$ at an equilibrium distance of 7.20 a.u.. This permanent dipole moment is among the strongest of all alkali-metal mixtures [39], making NaCs a good candidate for quantum computing. Photoassociation from an ultracold $\mathrm{Na}$ and $\mathrm{Cs}$ mixture can be used to obtain $\mathrm{NaCs}$ dimers at temperatures cold enough to be optically trapped [40]. Many other ultracold heteronuclear dimers have been obtained using various techniques [33]. Experimental techniques for trapping and manipulating ultracold molecules are rapidly developing [33]. We assume that the molecules can be trapped in the lowest translational state of succeeding sites of a three-dimensional optical lattice, with only one molecule per site and without tunneling from one site to another. Both the string of molecules and the electric field are aligned along the $Z$ axis of a laboratory-fixed frame chosen to be the quantization axis. The electric field orientates the molecules, resulting in a mixing of the rotational levels. The electric field may vary along the $Z$ axis to make the molecules individually addressable by spectroscopic means.

Molecules are initially stored in a high vibrational level of the ground $X^{1} \Sigma^{+}$potential. Information can be stored in these long-lived levels for they have no permanent dipole moment and the molecules can therefore be regarded as isolated from one to another even in the presence of the electric field. When a logical operation needs to be carried out, the required molecules (depending on the number of qubits for the computation) are brought to the lowest vibrational levels [35]. As opposed to before, these levels have a large dipole moment and the strong dipole-dipole interaction coupling adjacent molecules allow logic gate operations implemented on several molecules. One should emphasize that this procedure differs from the realization of logic gates based on switchable dipoledipole interactions proposed by Kuznetsova et al. [35] because, in our case, the variation of the dipole-dipole interaction is not part of the logical operation itself. For molecules separated by a distance $R$ of $300 \mathrm{~nm}$, the dipole-dipole interaction is of the order of a few tens of kilohertz. The interaction decreases rapidly as the distance between molecules increases due to its $1 / R^{3}$ variation. At $500 \mathrm{~nm}$ the interaction magnitude drops to a few hundreds of hertz. Desired driven transitions are differentiated from unwanted transitions by the dipole-dipole interaction. A small interaction will require a longer pulse duration in order to differentiate the transitions. The separation between molecules is dictated by the characteristics of the optical lattice the molecules will be loaded in. For $\mathrm{NaCs}$, a wavelength of $600 \mathrm{~nm}$ corresponds to a molecular transition above the $3 s+7 s$ asymptote and below the inner well of the ${ }^{1} \Sigma^{+}$potential correlating to the $3 s+7 p$ asymptote [41] in a region where overlap integrals with the lowest vibrational levels of the ground ${ }^{1} \Sigma^{+}$are extremely small, offering a favorable wavelength window for an optical trap with sites distant by a distance of $300 \mathrm{~nm}$. Similar features can be found for other heteronuclear alkali-metal molecules [42].

\section{B. Hamiltonian}

This work is restricted to the study of gates involving two or three qubits encoded in no more than two molecules. From now on, we use capital letters to refer to coordinates in the laboratory frame and lowercase letters when we refer to inner coordinates of the molecules. We denote $\left(\mathbf{e}_{X}, \mathbf{e}_{Y}, \mathbf{e}_{Z}\right)$ as the basis vector of a Cartesian space-fixed frame (or laboratory Cartesian frame). We denote $\mathbf{e}_{Q}, Q=0, \pm 1$, as the basis vector for a space-fixed spherical coordinate system. We denote $\left(\mathbf{e}_{x}, \mathbf{e}_{y}, \mathbf{e}_{z}\right)$ as the basis vector of a Cartesian molecular frame and $\mathbf{e}_{p}, p=0, \pm 1$, as a molecular spherical frame. When the spherical indices are ambiguous, we may use the index $R$ and $r$ instead of the index 0 for the laboratory and molecular frame, respectively.

We consider molecules in the ground $X^{1} \Sigma^{+}$electronic potential only. For $\mathrm{NaCs}$, we use the potential calculated as explained in [39]. The total Hamiltonian for two molecules can be written as the sum of a time-independent term, $H_{0}$, and 
a time-dependent perturbation term, $W(t)$ :

$$
H_{\text {tot }}(t)=H_{0}+W(t) .
$$

$W(t)$ represents the interaction of the molecules with a laser pulse polarized along the $\mathbf{e}_{Z}$ direction, $\mathbf{E}_{L}(t)=E_{L}(t) \cdot \mathbf{e}_{Z}$ :

$$
W(t)=\sum_{i}-\mu_{i} \cdot \mathbf{E}_{L}(t)=\sum_{i}-\mu_{i_{0}} E_{L}(t) \cos \theta_{i},
$$

where $\mu_{i_{0}}$ is the $z_{i}=r_{i}$ component of the dipole moment of molecule $i$ in the ground electronic state. $H_{0}$, which represents the energy of the molecules coupled by the dipole-dipole interaction, is made of several contributions:

$$
H_{0}=\sum_{i=1}^{2}\left(H_{\mathrm{ex}}^{i}+H_{\mathrm{in}}^{i}+H_{S}^{i}\right)+V_{\mathrm{dd}}
$$

$H_{\mathrm{ex}}^{i}, H_{\mathrm{in}}^{i}$, and $H_{S}^{i}$ are the external, internal, and Stark Hamiltonians associated with the molecule $i=1$ or 2 , respectively. $V_{\mathrm{dd}}$ is the dipole-dipole interaction.

The external Hamiltonian $H_{\mathrm{ex}}^{i}$ describes the motion of the molecule $i$ in the trapping optical potential. It can be approximated locally by a three-dimensional isotropical harmonic oscillator with frequency $\omega_{L}$ and depth $V_{0}$ [43]:

$$
H_{\mathrm{ex}}^{i}=\frac{\mathbf{P}_{i}^{2}}{2 M}+V_{\mathrm{opt}}\left(\mathbf{R}_{i}\right),
$$

where $M$ is the total mass of the molecule. $\omega_{L}$ and $V_{0}$ depend on the dynamic polarizabilities of the molecule and on the frequency and intensity of the trapping laser $[42,43]$. Typical values for $\omega_{L}$ are in kilohertz. The depth must be of the order of tens of kilohertz in order for the molecules to be tightly trapped and for the tunneling from one site to another to be negligible.

The inner Hamiltonian $H_{\mathrm{in}}^{i}$ describes an isolated diatomic molecule in the Born-Oppenheimer approximation. It contains the vibrational Hamiltonian $H_{\mathrm{vib}}^{i}$ in the ground $X^{1} \Sigma^{+}$ electronic state and the rotational Hamiltonian $H_{\text {rot }}^{i}$ :

$$
H_{\mathrm{in}}^{i}=H_{\mathrm{vib}}^{i}+H_{\mathrm{rot}}^{i}
$$

with

$$
H_{\mathrm{vib}}^{i}=\frac{\mathbf{p}_{i}^{2}}{2 m}+v\left(r_{i}\right),
$$

where $m$ is the reduced mass of the molecule vibrating in the interatomic potential $v\left(r_{i}\right), r_{i}$ being the interatomic coordinate.

For ${ }^{1} \Sigma^{+}$molecules, the rotational Hamiltonian for a rigid rotator is given by

$$
H_{\mathrm{rot}}^{i}=B_{v}^{i} \mathbf{N}^{2},
$$

where $\mathbf{N}$ is the total angular momentum and $B_{v}^{i}$ is the rotational constant in vibrational state $v$.

$H_{S}^{i}$ refers to the Stark Hamiltonian of a molecule in a static electric field $\mathbf{E}_{S}^{i}\left(\mathbf{R}_{i}\right)$. In our case, $\mathbf{E}_{S}^{i}\left(\mathbf{R}_{i}\right)$ is aligned along the $Z$ axis and varies only along this coordinate. The Stark Hamiltonian reduces to

$$
H_{S}^{i}=-E^{i}\left(Z_{i}\right) \mu_{i_{0}} \cos \left(\theta_{i}\right) .
$$

The last contribution comes from the long-range dipoledipole interaction term $V_{\mathrm{dd}}(\mathbf{R})$ of two molecules separated by a distance $\mathbf{R}=\mathbf{R}_{2}-\mathbf{R}_{1}=R e_{Z}=R e_{R}$ in the laboratory frame. By assuming that the molecules rotate in a plane, the projection of the rotational quantum number on the internuclear axis is conserved and the dipole-dipole interaction term becomes [36]

$$
V_{\mathrm{dd}}\left(R, \theta_{1} \theta_{2}\right)=-\frac{1}{2 \pi \epsilon_{0}} \frac{\mu_{0, i} \mu_{0, j}}{R^{3}} \cos \theta_{1} \cos \theta_{2},
$$

where the angles $\theta_{1}$ and $\theta_{2}$ characterize the orientation of the dipole moments versus the intermolecular axis $\mathbf{e}_{R}$.

\section{Product and coupled basis sets}

For a system of two interacting molecules, we define a product basis set $|\varphi\rangle$ constructed by tensorial product of the basis of the individual molecules:

$$
\begin{aligned}
|\varphi\rangle & =\left|n, v, N, m_{N}\right\rangle_{1} \otimes\left|n, v, N, m_{N}\right\rangle_{2}, \\
& =\left|n_{1}, n_{2}, v_{1}, N_{1}, m_{N_{1}}, v_{2}, N_{2}, m_{N_{2}}\right\rangle
\end{aligned}
$$

where the basis set $\left|n, v, N, m_{N}\right\rangle_{i}$ is built from a tensor product of the eigenbasis of $H_{\mathrm{ex}}^{i}, H_{\mathrm{vib}}^{i}$, and $H_{\mathrm{rot}}^{i}$.

We make the assumption that $V_{\mathrm{dd}}$ and $H_{S}$ only couple rotational levels. However, both terms can create couplings between states of the optical trap. For a molecule in the ground state of the trap, the translational ground-state wave function of the potential well is a Gaussian of width $a=\sqrt{\hbar / M \omega_{L}}$. For a typical experiment, $a$ is of the order of tens of micrometers. No coupling will occur if $H_{S}$ is constant over the range of variation of $a$, the coupling integral $\left\langle n_{i}\left|H_{S}\right| m_{i}\right\rangle$ involving the eigenfunctions of the harmonic trap being zero in this case. For the coupling due to the dipole-dipole interaction, a detailed analysis can be found in [35]. Coupling between translational states for typical polar molecules is small and results in an energy shift no larger than $1 \%$ of the unperturbed energy spacing. For a typical optical trap, where the spacing between translational levels is about tens of kilohertz, it gives a negligible shift of a few tens of hertz. The $n$ label can then be omitted in the basis function notation.

Also, we only consider motion in a plane $\phi_{i}=0$ with a field aligned along $\mathbf{e}_{Z}$ so only $m_{N}=0$ states are involved. Finally, the relevant contracted notation $\left|v_{1}, N_{1}, v_{2}, N_{2}\right\rangle$ will label the states of the total product basis. It can be further simplified to just $\left|N_{1}, N_{2}\right\rangle$ when only one vibrational state is considered.

The eigenstates $|\tilde{\varphi}\rangle$ that diagonalize the total timeindependent Hamiltonian $H_{0}$ can be written as a superposition of the initial product basis states with different amplitudes $p_{k}$ :

$$
|\tilde{\varphi}\rangle=\sum_{k} p_{k}\left|v_{1}, N_{1}, v_{2}, N_{2}\right\rangle_{k} \equiv\left|\tilde{v}_{1}, \tilde{N}_{1}, \tilde{v}_{2}, \tilde{N}_{2}\right\rangle,
$$

where the tilde notation $|\tilde{\varphi}\rangle=\left|\tilde{v}_{1}, \tilde{N}_{1}, \tilde{v}_{2}, \tilde{N}_{2}\right\rangle$ helps in identifying the main component to which they are adiabatically connected. When the vibrational manifold is well known, the tilde notation can be further reduced to just $|\tilde{\varphi}\rangle=\left|\tilde{N}_{1}, \tilde{N}_{2}\right\rangle$. The state mixing allows electric dipole transitions between different $\left|\tilde{v}_{1}, \tilde{N}_{1}, \tilde{v}_{2}, \tilde{N}_{2}\right\rangle$ states even if the transition would be forbidden by considering the initial $\left|v_{1}, N_{1}, v_{2}, N_{2}\right\rangle$ states. 


\section{Dynamics}

The time-dependent Schrödinger equation will be solved in the coupled basis set in the interaction representation [44]. The evolutions of the populations $b_{n}(t)$ are given by the set of coupled equations:

$$
i \hbar \frac{d}{d t} b_{n}(t)=\sum_{k} e^{i \omega_{n k} t} W_{n k}(t) b_{k}(t),
$$

where we have introduced the Bohr pulsation $\omega_{n k}=\left(E_{n}-\right.$ $\left.E_{k}\right) / \hbar$ and the matrix elements $W_{n k}(t)$ of the time-dependent interaction in the eigenbasis of $H_{0}$ :

$$
W_{n k}(t)=\left\langle\tilde{\varphi}_{n}|W| \tilde{\varphi}_{k}\right\rangle .
$$

The coupled equations are solved using the fourth-order Runge-Kutta method [45] without any rotating wave approximation.

\section{INTERMOLECULAR CNOT GATE}

We first examine the possibility of steering elementary gates by $\pi$ pulses in the microwave and infrared domains by focusing on conditional population inversions. The control of the phase is considered in the final section.

The first example concerns the two-qubit controlled-NOT (CNOT) gate which flips the second (target) qubit if the first one (control qubit) is equal to 1 :

$$
\begin{aligned}
& (\alpha|00\rangle+\beta|01\rangle+\gamma|10\rangle+\delta|11\rangle) \\
& \quad \stackrel{\mathrm{CNOT}}{\longrightarrow}(\alpha|00\rangle+\beta|01\rangle+\gamma|11\rangle+\delta|10\rangle) .
\end{aligned}
$$

The gate is driven by a $\pi$ pulse defined by

$$
E_{\pi}(t)=E_{\pi}^{0} \sin ^{2}\left(\frac{\pi t}{\tau_{p}}\right) \cos \left(\omega_{i f} t\right),
$$

with amplitude $E_{\pi}^{0}=\frac{2 \pi}{\tau_{p} \mu_{i f}}$, where $\tau_{p}$ is the duration of the pulse and $\mu_{i f}$ is the dipole moment for the transition. The scheme is similar to some early proposals for conditional quantum dynamics $[46,47]$. It uses the fact that a transition frequency in the second molecule depends on the state of the first molecule.

The qubits are encoded in two neighboring molecules. Both molecules are in the ground vibrational states $\tilde{v}_{1}=\tilde{v}_{2}=0$ so that the states are denoted only by the rotational states $\left|\tilde{N}_{i}, \tilde{N}_{j}\right\rangle$. The logical states are encoded into the first four states of the coupled basis set which correlate with the $N_{1}=0$ or 1 and $N_{2}=0$ or 1 states (see Fig. 2). In the product basis the CNOT transformation should consist in flipping the rotational state of the second molecule if and only if the first one is in state $N_{1}=1$. In a basis set diagonalizing the Stark Hamiltonian without dipole-dipole interaction, the frequencies of the rotational transitions in the second molecule when the first one is in the state $N_{1}=0\left(\omega_{0}\right)$ or $N_{1}=1\left(\omega_{1}\right)$ are the same (see Fig. 2). The dipole-dipole interaction leads to two very close but different frequencies. This allows us to excite only the transition $|\tilde{1} \tilde{0}\rangle \rightarrow|\tilde{1} \tilde{1}\rangle$ at a frequency $\tilde{\omega}_{1}$ (solid red arrow in Fig. 2) and not the transition $|\tilde{0} \tilde{0}\rangle \rightarrow|\tilde{0} \tilde{1}\rangle$ at the frequency $\tilde{\omega}_{0}$ (dashed red arrow). When the system is in a computational basis state, a $\pi$ pulse can selectively drive the desired population inversion. (a)

(b)

(c)

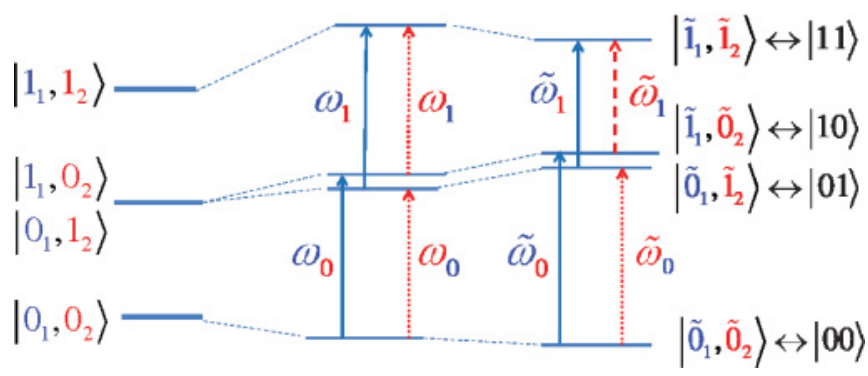

FIG. 2. (Color online) Diagram of the lowest eigenvalues of the total time-independent Hamiltonian for three different cases: (a) without any electric field, (b) with electric fields $E^{1}>E^{2}$ without dipole-dipole interaction, and (c) same as (b) plus the dipole-dipole interaction. The eigenvectors $\left|\tilde{N}_{1}, \tilde{N}_{2}\right\rangle$ correlate adiabatically to the vector $\left|N_{1}, N_{2}\right\rangle$ with $v_{1}=v_{2}=0$. Only $m_{N}=0$ are considered. The $m_{N}$ and $v_{i}$ labels are omitted. Transitions in the first or second molecule are shown by solid blue or dotted red arrows, respectively. The dashed red arrow indicates the transition for the realization of a CNOT operation. The subscripts labeling the frequencies indicate the rotational state of the neighboring molecule.

Due to the presence of transitions with close frequencies, the overall gate fidelity can be altered by a loss of population to levels reached by off-resonance unwanted transitions. Pulse durations are determined in order to avoid them. This can be done by simply increasing the pulse duration in the numerical simulations until numerical results show that perturbing transitions do not occur. This tedious numerical checking procedure can be facilitated by a preceding analytical analysis. The Rosen-Zener formula [48,49] gives the transition probability $P$ as a function of the pulse detuning $\Delta$; assuming a hyperbolic secant pulse envelop of width $T_{\mathrm{RZ}}$ centered at $t_{0}, f_{\mathrm{RZ}}(t)=\operatorname{sech}\left[\left(t-t_{0}\right) / T_{\mathrm{RZ}}\right]$, we have

$$
P=\frac{\sin ^{2}\left(\frac{1}{2} \pi \mu_{i f} E_{0} T_{\mathrm{RZ}}\right)}{\cosh ^{2}\left(\frac{1}{2} \pi \Delta T_{\mathrm{RZ}}\right)},
$$

with $E_{0}$ being the amplitude of the field and $\mu_{i f}$ the dipole moment of the transition. the $T_{\mathrm{RZ}}$ parameter is fitted to make the analytical pulse similar to the sine-square pulse used numerically $\left(f_{\sin }(t)=\sin ^{2}\left[(\pi t) / \tau_{p}\right]\right)$. Taking $\tau_{p}=\beta T_{\mathrm{RZ}}$ with $\beta=7$ leads to good results. Using the probability formula, we established that the pulse duration $\tau_{p}$, to avoid an off-resonance transition characterized by a frequency difference $\Delta$, must satisfy the condition $\tau_{p}>10 \hbar / \Delta$.

Figure 3 shows the evolution of the populations during the CNOT gate driven by a $\pi$ pulse starting from the four different states of the computational basis. The states corresponding to the logical states $|00\rangle$ and $|01\rangle$ temporarily leave the computational space but correctly return to it with an accumulated phase (the phase constraint is discussed later). From now on, simulations are run with the following fixed parameters: $R_{12}=300 \mathrm{~nm}, E^{1}=2 \mathrm{kV} \mathrm{cm}^{-1}$, and $E^{2}=$ $1.5 \mathrm{kV} \mathrm{cm}^{-1}$. The dynamical basis set for the simulation contains the vibrational state $v=0$ and rotational states up to $N=4$ for each molecule. The difference of frequencies which must be resolved is $4.002 \times 10^{-6} \mathrm{~cm}^{-1}$ and the pulse 

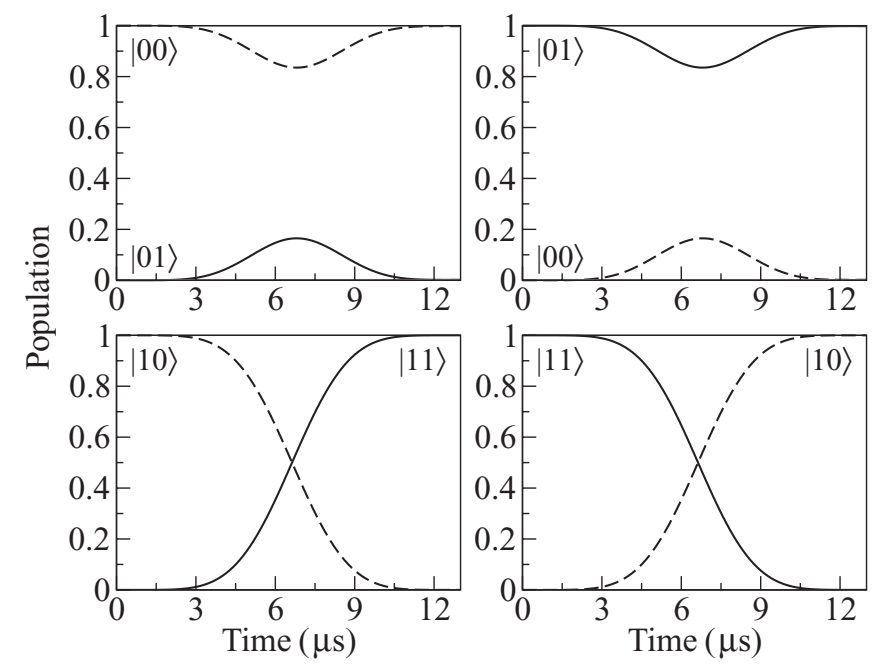

FIG. 3. Evolution of the populations during the CNOT gate driven by a $\pi$ pulse. When the control qubit is 0 (initial states $|00\rangle$ or $|01\rangle$ ), the final state of the qubits remains unchanged when the pulse is applied, although the population may vary during the process (top left and right panels, respectively). On the contrary, the second qubit is flipped when the initial state is $|10\rangle$ or $|11\rangle$ leading to final states $|11\rangle$ or $|10\rangle$ (bottom left and right panels, respectively).

duration must be at least $\tau_{p}=13.2 \mu \mathrm{s}$. The intensity of the $\pi$ pulse is $E_{\pi}^{0}=7.5 \mathrm{~V} \mathrm{~m}^{-1}$.

Figure 4 shows the product state mixing due to the static electric field for the four eigenstates of the time-independent Hamiltonian we used to encode the qubits. The square of the weight coefficients $\left|p_{k}\right|^{2}$ ( $y$ axis) are plotted as functions of the different state product basis functions $\left|N_{1}, N_{2}\right\rangle$ ( $x$ axis). For the range of electric field considered in this work, eigenstates have one primary component accounting for about $60 \%$. The electric field introduces important mixing between adjacent levels with $\Delta N= \pm 1$. Although the mixing between levels with $\Delta N= \pm 2$ or $N= \pm 3$ is weaker, it is not negligible and it allows electric dipole transitions to levels that would be forbidden by usual selection rules in the absence of an electric field. One example is the transition $v_{1}=0$ and $J_{1}=1$ to $v_{1}=2$ and $J_{1}=3$ involved in a CNOT gate introduced in the next section. The assignment of the tilde rotational quantum states and the choices of favorable transitions require a careful analysis of the state decomposition and of the dipole matrix elements expressed in the eigenbasis of the time-independent Hamiltonian $H_{0}$.

\section{0 AND 1 ADDER}

The adder of 0 and 1 (0-ADD and 1-ADD) [37] is a simplification of the more general full adder of two binary digits $a_{i}$ and $b_{i}$ and a carry in $c_{i}$ [50]. Similar to the classical case, the addition of two numbers in binary representation, $a=\left(a_{1} a_{2} \ldots a_{i} \ldots a_{n}\right)$ and $b=\left(b_{1} b_{2} \ldots b_{i} \ldots b_{n}\right)$, is performed digit by digit starting from the least significant one. For the $i$ th rank, one needs to evaluate a sum $s_{i}$ and a carry out $c_{i+1}$. A cycle of the quantum full adder requires four qubits [50,51]. However, in the special case of a 0 adder or a 1 adder, only three qubits operations are necessary. The $c_{i}$ and $b_{i}$ digits are encoded in the first two qubits and the third is 0 . One has

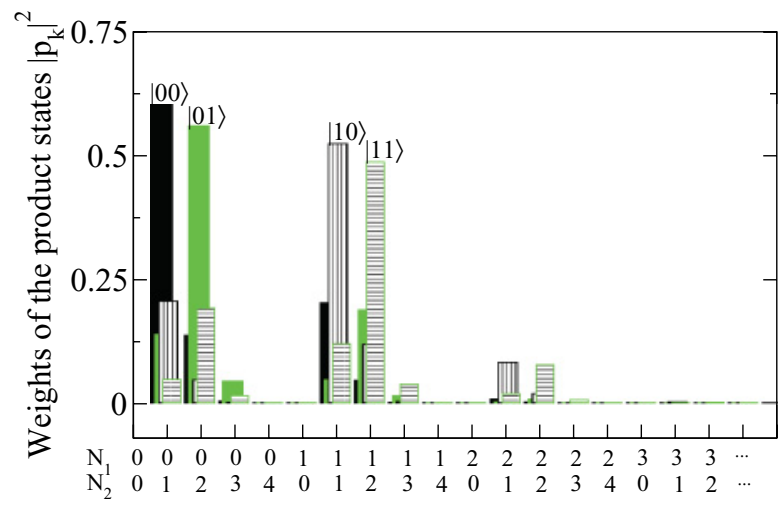

FIG. 4. (Color online) Weights $\left|p_{k}\right|^{2}$ of the product basis states $\left|N_{1}, N_{2}\right\rangle$ for the four lowest eigenvectors of the time-independent Hamiltonian showing the state mixing due to the static Stark electric field and the dipole-dipole coupling in the manifold $v=0$. The state mixture is similar in other $v$. Eigenstates are labeled after the main component of the product basis. Qubits $|10\rangle$ and $|11\rangle$ encoded in eigenstates $\left|\tilde{N}_{1}=1, \tilde{N}_{2}=0\right\rangle$ and $\left|\tilde{N}_{1}=1, \tilde{N}_{2}=1\right\rangle$, respectively, have a non-negligeable admixture of states with $N_{i}=3$ allowing electric dipole transitions to these states.

initially $\left|Q_{1} Q_{2} Q_{3}\right\rangle=\left|c_{i}, b_{i}, 0\right\rangle$. The information that $a_{i}$ is 0 or 1 , respectively, is contained in the applied pulse, which is different for the 0-ADD or 1-ADD gate. The results $s_{i}$ and $c_{i+1}$ are stored in the second and third qubits, respectively. One has finally $\left|Q_{1} Q_{2} Q_{3}\right\rangle=\left|c_{i}, s_{i}, c_{i+1}\right\rangle$. The 0-ADD or 1-ADD that drives the unitary transformation $\left|c_{i}, b_{i}, 0\right\rangle \rightarrow\left|c_{i}, s_{i}, c_{i+1}\right\rangle$ for $a_{i}$ is 0 or 1 , respectively. The truth tables are given in Table I (note that only the first four inputs with $Q_{3}=0$ are effectively used; the last four inputs ensure the reversibility of the operations).

As schematized in Fig. 5, the carry out of the 0-ADD gate is $c_{i}$.AND. $b_{i}$ and is computed by the three-qubit TOFFOLI gate: $|x\rangle|y\rangle|z\rangle \rightarrow|x\rangle|y\rangle|z \oplus x y\rangle$, where $\oplus$ is the sum modulo 2 . The TOFFOLI gate corresponds to the AND gate when the third qubit is zero. It is the controlled-controlled NOT which flips the third qubit if the first two qubits are in state 1 . The sum

TABLE I. 0 adder and 1 adder truth tables. The information $a_{i}=0$ and $a_{i}=1$, respectively, is contained in the pulse driving the transformation. Only the first four inputs with $Q_{3}=0$ are effectively used. $\bar{c}_{i+1}=1 \oplus c_{i+1}$.

\begin{tabular}{|c|c|c|c|c|c|c|c|c|c|c|c|}
\hline \multicolumn{6}{|c|}{0 adder } & \multicolumn{6}{|c|}{1 adder } \\
\hline$c_{i}$ & $b_{i}$ & $Q_{3}$ & $c_{i}$ & $s_{i}$ & $c_{i+1}$ & $c_{i}$ & $b_{i}$ & $Q_{3}$ & $c_{i}$ & $s_{i}$ & $c_{i+1}$ \\
\hline 0 & 0 & 0 & 0 & 0 & 0 & 0 & 0 & 0 & 0 & 1 & 0 \\
\hline 0 & 1 & 0 & 0 & 1 & 0 & 0 & 1 & 0 & 0 & 0 & 1 \\
\hline 1 & 0 & 0 & 1 & 1 & 0 & 1 & 0 & 0 & 1 & 0 & 1 \\
\hline 1 & 1 & 0 & 1 & 0 & 1 & 1 & 1 & 0 & 1 & 1 & 1 \\
\hline$c_{i}$ & $b_{i}$ & $Q_{3}$ & $c_{i}$ & $s_{i}$ & $\bar{c}_{i+1}$ & $c_{i}$ & $b_{i}$ & $Q_{3}$ & $c_{i}$ & $s_{i}$ & $\bar{c}_{i+1}$ \\
\hline 0 & 0 & 1 & 0 & 0 & 1 & 0 & 0 & 1 & 0 & 1 & 1 \\
\hline 0 & 1 & 1 & 0 & 1 & 1 & 0 & 1 & 1 & 0 & 0 & 0 \\
\hline 1 & 0 & 1 & 1 & 1 & 1 & 1 & 0 & 1 & 1 & 0 & 0 \\
\hline 1 & 1 & 1 & 1 & 0 & 0 & 1 & 1 & 1 & 1 & 1 & 0 \\
\hline
\end{tabular}




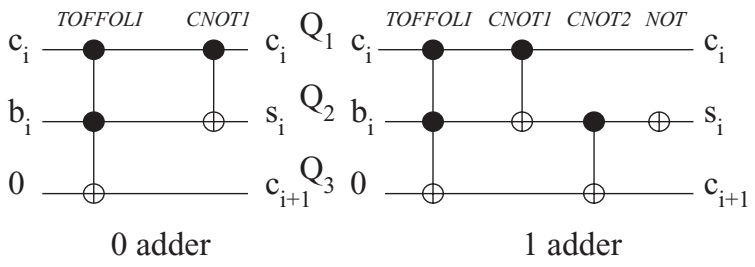

FIG. 5. Logical circuits for the 0 adder (left panel) and 1 adder (right panel).

$s_{i}$ is given by the first CNOT gate (noted CNOT1): $|x\rangle|y\rangle \rightarrow$ $|x\rangle|y \oplus x\rangle$. The 1-ADD is the continuation of the 0-ADD. For the 1-ADD, we can see from Table I that $s_{i}$ is obtained from the $s_{i}$ of the 0 -ADD by an additional NOT gate which simply flips the state $(|0\rangle$ and $|1\rangle)$ of the qubit. It can be seen also that $c_{i+1}$ of the 1-ADD only needs to do the sum modulo 2 of the $s_{i}$ and the carry out $c_{i+1}$ of the 0 -ADD which are encoded in the second and third qubits. As a result this step is a new CNOT (noted CNOT2) with control qubit $Q_{2}$ and target qubit $Q_{3}$.

The most promising implementation of the 0 -ADD and 1-ADD consists in encoding the carry $c_{i}$ and the number $b_{i}$ in the rotational and the vibrational structure of the first molecule, whereas the carry out $c_{i+1}$ is encoded in the rotation of the second molecule. So one has $\left|Q_{1} Q_{2} Q_{3}\right\rangle=\left|\tilde{N}_{1}, \tilde{v}_{1}, \tilde{N}_{2}\right\rangle$. The sum $s_{i}$ for the $i$ th step of the addition will be stored in the vibration of the first molecule in replacement of $b_{i}$ and the carry out encoded in the second molecule becomes the carry in for the $(i+1)$ th step allowing the addition to be further continued.

When the logical states are mapped on a coupled basis set and not on a product basis set, one has to steer the transformation in the total computational basis set (a three-qubit space here). For a one-qubit (or two-qubit) transformation one has to consider all the transformations involving the active qubit(s) in the three-qubit space. For example, the extended operator of the NOT gate on $Q_{2}$ is

$$
\tilde{U}_{Q_{2}}=E_{Q_{1}} \otimes U_{Q_{2}} \otimes E_{Q_{3}},
$$

where $E_{Q_{k}}$ is the unity matrix in the one-qubit space $k$. This leads to four population inversions in place of a single one in a product basis set because the $Q_{2}$ flip must be realized for each possible value of $Q_{1}$ and $Q_{3}$. Second, one should point out that whatever the encoding is, unwanted transitions can always occur. The length of the pulses are then chosen to satisfy two opposite conditions. The pulse must be short enough to simultaneously drive a maximum number of active transitions with a single carrier frequency and it must be long enough to avoid these unwanted transitions whose frequencies are very close.

\section{A. Intermolecular TOFFOLI gate}

Conditional dynamics are more demanding for the TOFFOLI gate than for the CNOT gate presented in the previous section. Although the computational basis $\left|Q_{1} Q_{2} Q_{3}\right\rangle$ generated by the eight combinations of $\tilde{N}_{1}=0$ or $1, \tilde{v}_{1}=0$ or $1, \tilde{N}_{2}=0$ or 1 (with $\tilde{v}_{2}$ always equal to 0 ) would provide an intuitive mapping between the quantum numbers and the logical states, it is rather inconvenient in practice because of the very similar rotational

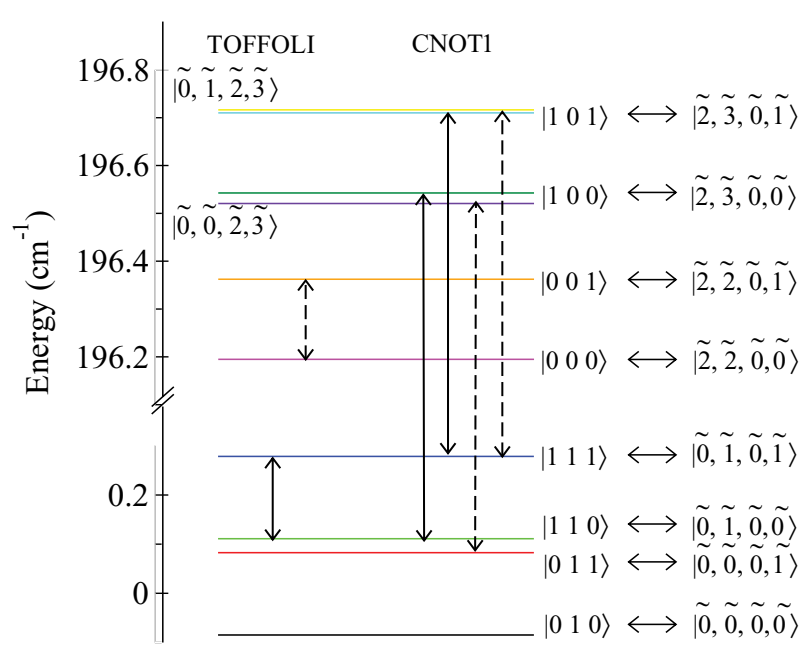

FIG. 6. (Color online) Encoding of the three qubits for the 0 adder in the states of the coupled basis set $\left|Q_{1} Q_{2} Q_{3}\right\rangle \leftrightarrow\left|\tilde{v}_{1}, \tilde{N}_{1}, \tilde{v}_{2}, \tilde{N}_{2}\right\rangle$. The active transitions for the TOFFOLI and CNOT gates (the latter is called CNOT1 in the text) are shown by solid arrows and the corresponding unwanted transitions are shown by dashed arrows. The zero of energy is chosen at the ground state without Stark field and dipole-dipole coupling.

constants for the two vibrational levels. With this assignment, the rotational state of the second molecule must be flipped if and only if the first molecule is in a given rotational and vibrational state. Then, except for $\tilde{N}_{2}$ holding the carry out, we adopt an assignment with no correspondance between the quantum numbers and the associated qubit state. The eight states and the logical mapping are shown in Fig. 6. In the present case, the frequency of the $|110\rangle \leftrightarrow|111\rangle$ TOFFOLI transition (solid arrow in Fig. 6) is fairly close to the unwanted $|000\rangle \leftrightarrow|001\rangle$ transition. The difference in frequency which must be resolved is then $4.0 \times 10^{-7} \mathrm{~cm}^{-1}$.

The population of each computational basis state during the TOFFOLI gate is plotted in the top panel of Fig. 7. Each population is weighted by a factor different from 1 for clarity (it is not a superposed state). The duration of the $\pi$ pulse is $\tau_{p}=253 \mu \mathrm{s}$. The carrier frequency is in resonance with the active transition $|110\rangle \leftrightarrow|111\rangle$, that is, $|\tilde{0}, \tilde{1}, \tilde{0}, \tilde{0}\rangle \leftrightarrow|\tilde{0}, \tilde{1}, \tilde{0}, \tilde{1}\rangle$ with $\omega=0.16743 \mathrm{~cm}^{-1}$. The amplitude of the pulse is $E_{0}=$ $4.1506 \times 10^{-6} \mathrm{kV} / \mathrm{cm}$. For all the simulations relative to the adder algorithm, the dynamical basis set contains 64 states including the $v_{2}=2$ manifold $\left(v_{1}=v_{2}=0\right.$ with $J_{1}$ and $J_{2}=$ $0,1,2 ; v_{1}=v_{2}=2$ with $\left.J_{1}=0,1,2,3,4\right)$.

The population inversion is very good with a fidelity of 0.9999 . Note that the $\pi$ pulse is not optimized for a superposition but is sufficient for arithmetic operations for which the system is always in a computational basis state. As illustrated in the next section, optimization for any superposition can be obtained with OCT.

\section{B. Intramolecular CNOT1 gate}

This gate is intramolecular since both the control $Q_{1}$ and target $Q_{2}$ qubits are encoded in the first molecule (the rotation and the vibration, respectively). The extended CNOT1 gate involves the following transitions: $\left|10 Q_{3}\right\rangle \leftrightarrow\left|11 Q_{3}\right\rangle$ for any 


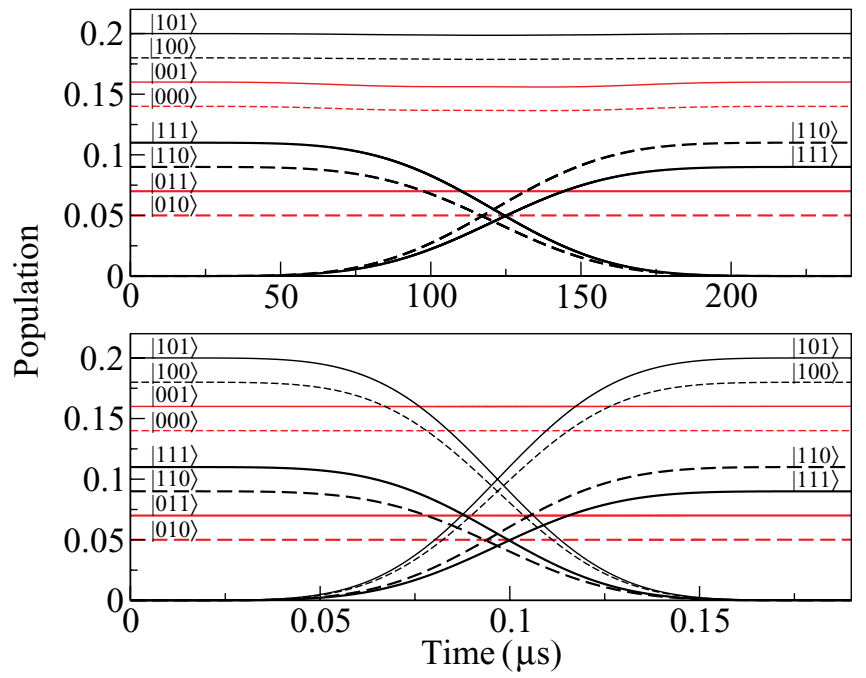

FIG. 7. (Color online) Population evolution for each computational basis state showing the realization of the gates of the 0 adder. Upper panel: TOFFOLI gate. Lower panel: CNOT1 gate. For both panels, simulations were performed considering an initial population of 1 in one single pure computational basis state at a time. For a better reading of the picture, populations have been weighted by different arbitrary factors. The picture does not present the evolution of the population for an arbitrary superposition of computational basis states. Note that the time scale is different from one panel to the other.

value of the third qubit $Q_{3}$. These two transitions are shown with solid arrows in Fig. 6:

$$
\begin{aligned}
& \omega_{Q_{3}=0}=|100\rangle \equiv|\tilde{2} \tilde{3} \tilde{0} \tilde{0}\rangle \leftrightarrow|110\rangle \equiv|\tilde{0} \tilde{1} \tilde{0} \tilde{0}\rangle, \\
& \omega_{Q_{3}=1}=|101\rangle \equiv|\tilde{2} \tilde{3} \tilde{0} \tilde{1}\rangle \leftrightarrow|111\rangle \equiv|\tilde{0} \tilde{1} \tilde{0} \tilde{1}\rangle .
\end{aligned}
$$

They correspond to vibrational transitions in the first molecule and thus belong to the infrared domain. This is an example for which the electric dipole transition becomes possible only due to the state mixing since the transition from $v_{1}=0$ and $J_{1}=1$ to $v_{1}=2$ and $J_{1}=3$ is forbidden between the product basis states. The transition moment is weak $d=-4 \cdot 310^{-5}$ a.u. but yet allows reasonable field amplitude. The two transitions being very close $\left(\Delta \omega=8.3 \times 10^{-7} \mathrm{~cm}^{-1}\right)$, they can be induced by a single $\pi$ pulse. Two unwanted frequencies are shown by dashed arrows in Fig. 6. They involve vibrational states of the second molecule. Fortunately, these transitions differ from the active transitions by about $6 \times 10^{-3} \mathrm{~cm}^{-1}$. A shorter pulse than for the TOFFOLI gate can be used.

The botton panel of Fig. 7 shows the populations of each computational basis state during the CNOT1 gate. The pulse duration is $\tau_{p}=0.194 \mu \mathrm{s}$. The carrier frequency is fixed by the $|100\rangle \leftrightarrow|110\rangle$ transition and is equal to $\omega_{Q_{3}=0}=$ $196.43 \mathrm{~cm}^{-1}$. The amplitude is $E_{0}=93.793 \mathrm{kV} / \mathrm{cm}$.

\section{Intermolecular CNOT2 gate}

This logical operation is $\left|1 Q_{2} 0\right\rangle \leftrightarrow\left|1 Q_{2} 1\right\rangle$ for any value of the second qubit $Q_{2}$. We then have two active transitions:

$$
\begin{aligned}
\omega_{Q_{2}=0} & =|100\rangle \equiv|\tilde{2} \tilde{3} \tilde{0} \tilde{0}\rangle \leftrightarrow|101\rangle \equiv \mid 2 \tilde{3} \tilde{3} \tilde{1} \tilde{}), \\
\omega_{Q_{2}=1} & =|110\rangle \equiv|\tilde{0} \tilde{1} \tilde{0} \tilde{0}\rangle \leftrightarrow|111\rangle \equiv|\tilde{0} \tilde{1} \tilde{0} \tilde{1}\rangle .
\end{aligned}
$$

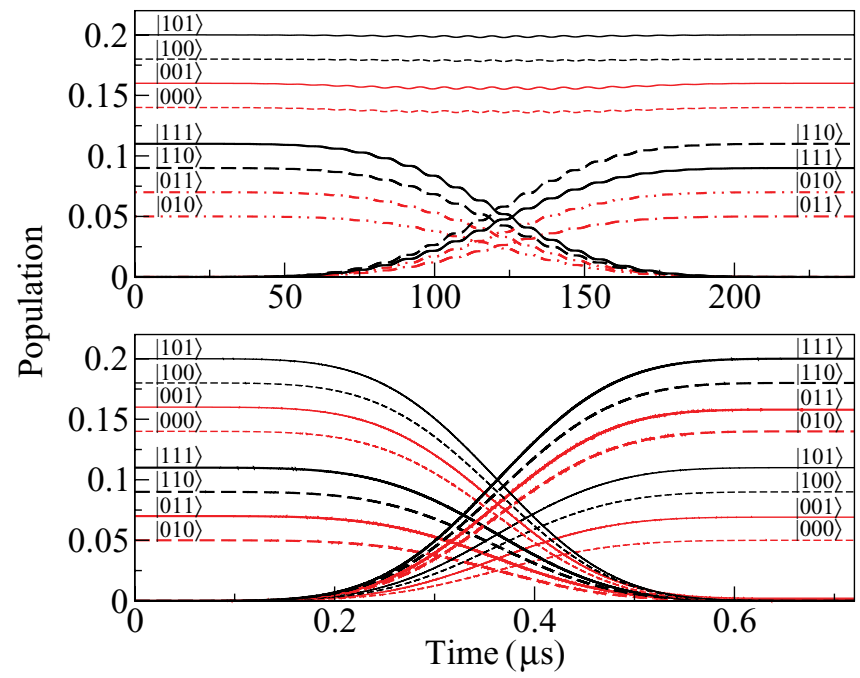

FIG. 8. (Color online) Population evolution of each computational basis state during the supplementary gates of the 1 adder. Upper panel: CNOT2 gate. Lower panel: NOT gate. For both panels, simulations were performed considering an initial population of 1 in one single pure computational basis state at a time. For a better reading of the picture, populations have been weighted by different arbitrary factors. The picture does not present the evolution of the population for an arbitrary superposition of computational basis states. Note that the time scale is different from one panel to the other.

The top panel of Fig. 8 shows the evolution of populations corresponding to the intermolecular CNOT gate (named CNOT2). In this case, we cannot use a unique pulse frequency for both transitions. A superposition of two microwave $\pi$ pulses are used. As for the TOFFOLI gate, we need to resolve a frequency difference of $4 \times 10^{-7} \mathrm{~cm}^{-1}$. The parameters for the pulses are $\tau_{p}=242 \mu \mathrm{s}, \omega_{Q_{2}=0}=0.16734 \mathrm{~cm}^{-1}$, and $E_{0}=4.1509 \times$ $10^{-6} \mathrm{kV} / \mathrm{cm}$ and $\omega_{Q_{2=1}}=0.16744 \mathrm{~cm}^{-1}$ and $E_{0}=4.1508 \times$ $10^{-6} \mathrm{kV} / \mathrm{cm}$.

\section{Intramolecular NOT gate}

The frequencies of the four active transitions of the extended NOT gate $\left(\left|Q_{1} 0 Q_{3}\right\rangle \leftrightarrow\left|Q_{1} 1 Q_{3}\right\rangle\right.$ for any values of $Q_{1}$ and $Q_{3}$ ) are

$$
\begin{aligned}
& \omega_{Q_{1}=0, Q_{3}=0}=|000\rangle \equiv|\tilde{2} \tilde{2} \tilde{0} \tilde{0}\rangle \leftrightarrow|000\rangle \equiv|\tilde{0} \tilde{0} \tilde{0} \tilde{0}\rangle, \\
& \omega_{Q_{1}=1, Q_{3}=0}=|100\rangle \equiv|\tilde{2} \tilde{3} \tilde{0} \tilde{0}\rangle \leftrightarrow|110\rangle \equiv|\tilde{0} \tilde{1} \tilde{0} \tilde{0}\rangle, \\
& \omega_{Q_{1}=0, Q_{3}=1}=|001\rangle \equiv|\tilde{2} \tilde{2} \tilde{0} \tilde{1}\rangle \leftrightarrow|011\rangle \equiv|\tilde{0} \tilde{0} \tilde{0} \tilde{1}\rangle, \\
& \omega_{Q_{1}=1, Q_{3}=1}=|101\rangle \equiv|\tilde{2} \tilde{3} \tilde{0} \tilde{1}\rangle \leftrightarrow|111\rangle \equiv|\tilde{0} \tilde{1} \tilde{0} \tilde{1}\rangle .
\end{aligned}
$$

The bottom panel of Fig. 8 shows the population evolutions for the final NOT gate.

The frequencies $\omega_{Q_{1}=0, Q_{3}=0}$ and $\omega_{Q_{1}=1, Q_{3}=0}$ on one side and $\omega_{Q_{1}=0, Q_{3}=1}$ and $\omega_{Q_{1}=1, Q_{3}=1}$ on the other side are sufficiently close to be driven by a single $\pi$ pulse $\left(\Delta \omega=4 \times 10^{-6} \mathrm{~cm}^{-1}\right.$ and $\Delta \omega=8 \times 10^{-7} \mathrm{~cm}^{-1}$, respectively). The two pulses have a duration adapted to avoid unwanted transitions $(\Delta \omega=6 \times$ $\left.10^{-3} \mathrm{~cm}^{-1}\right), \tau_{p}=0.726 \mu \mathrm{s}$. The frequencies are $\omega_{Q_{1}=0, Q_{3}=0}=$ $196.28 \mathrm{~cm}^{-1}$ and $\omega_{Q_{1}=1, Q_{3}=0}=196.43 \mathrm{~cm}^{-1}$. The amplitudes are $E_{0}=25.0115 \mathrm{kV} / \mathrm{cm}$ and $E_{0}=14.4558 \mathrm{kV} / \mathrm{cm}$. 


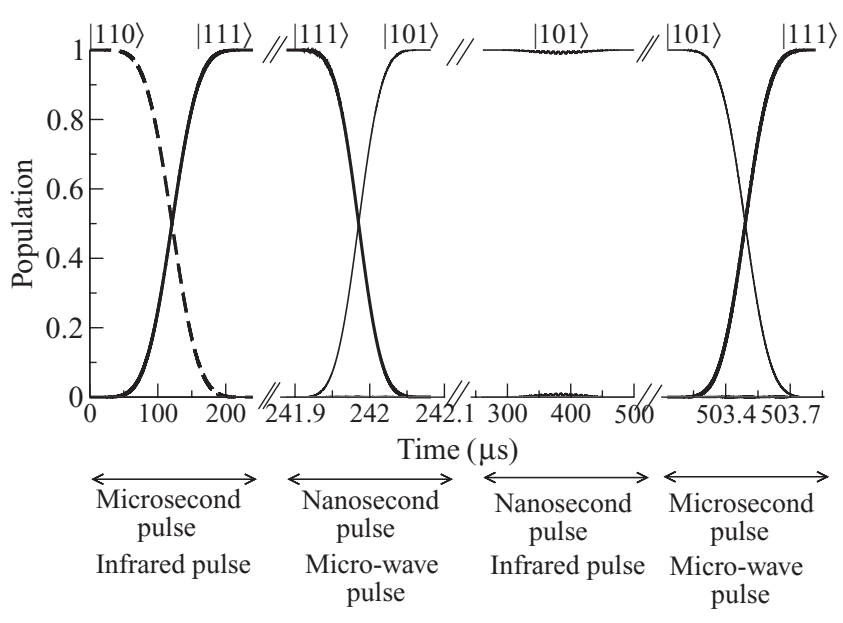

FIG. 9. Population evolution of the computational basis state corresponding to the logical state $|110\rangle\left(b_{i}=1\right.$ and $c_{i}=1$ during the 1-ADD gate (TOFFOLI-CNOT1-CNOT2-NOT gates) to give the final logical state $|111\rangle$ corresponding to $s_{i}=1$ and $c_{i+1}=1$.

\section{E. Intramolecular initialization}

Finally, one has to discuss the initialization of an addition cycle. The digit $b_{i}$ must be encoded in the first molecule for any value of the carry in $c_{i}$ which is unknown. With the assignment used here (see Fig. 6), the digit is encoded in the vibration of the first molecule. If $b_{i}=1$, the molecule must be in the manifold $\tilde{v}_{1}=0$. This means that the system is directly ready since the algorithm generates the carry out in this ground vibrational state. If $b_{i}=0$, the molecule must be in the manifold $\tilde{v}_{1}=2$. Two transitions must then be driven:

$$
\begin{aligned}
& |010\rangle \equiv|\tilde{0} \tilde{0} \tilde{0} \tilde{0}\rangle \rightarrow|000\rangle \equiv|\tilde{2} \tilde{2} \tilde{0} \tilde{0}\rangle, \\
& |110\rangle \equiv|\tilde{0} \tilde{1} \tilde{0} \tilde{0}\rangle \rightarrow|100\rangle \equiv|\tilde{2} \tilde{3} \tilde{0} \tilde{0}\rangle .
\end{aligned}
$$

They correspond to two transitions with frequencies $\omega_{Q_{1}=0, Q_{3}=0}$ and $\omega_{Q_{1}=1, Q_{3}=0}$ of the NOT gate realized previously and the NOT pulse can then be used to initialize the addition cycle.

\section{F. Concatenation}

Figure 9 illustrates the entire 1-ADD operation for the example $b_{i}=1$ and $c_{i}=1$. The initial logical state is $|110\rangle$. The network TOFFOLI (intermolecular), CNOT1 (intramolecular), CNOT2 (intermolecular), and NOT (intramolecular) gates operating in the microwave or infrared domain drives the system toward the final $|111\rangle$ logical state corresponding to $s_{i}=1$ and $c_{i+1}=1$.

\section{G. Discussion}

Any perturbation to the energy level structure coming from neighboring molecules, hyperfine interactions, coupling with the translational states in the lattice will drastically affect the high-fidelity logical operations the pulses were designed to drive.

We chose the electric field to be very different from one molecule to the other $(2 \mathrm{kV} / \mathrm{cm}$ and $1.5 \mathrm{kV} / \mathrm{cm}$ in our simulations). Significantly different electric fields lead to well differentiated energy structures on each molecule allowing fast intramolecular gates (implemented on single molecules). Having such a large variation of the electric field may generate couplings between translational states of the trapping potential because the requirement of a constant field over the range of variation of $a$ might be experimentally challenging to achieve. A much smoother gradient might prove to be more suitable to this respect. We tried simulations with different gradients and it was always possible to drive algorithms although intramolecular gate times had to be longer when the gradient was small $\left(E^{1}=2 \mathrm{kV} / \mathrm{cm}\right.$ and $\left.E^{2}=1.9 \mathrm{kV} / \mathrm{cm}\right)$. On the contrary, the time scale for intermolecular gate remains the same being mainly fixed by the dipole-dipole interaction.

We assumed that the electric field was orientated along the intermolecular axis. Note that results will remain valid for other field orientations. Only the expression of the interaction will differ from what we presented in this model, but the order of magnitude of the interaction will be about the same.

The effect of neighboring molecules interacting with the two-molecule systems has been evaluated. We have analyzed the energy shifts on the levels of the active molecules $j$ and $j+1$ by adding two molecules (at positions $j-1$ and $j+2$ ) and using the same gradient for the Stark field as for our simulations. The basis set is composed of manifolds $v=0$ with $N=0,1$, or 2 and $v=2$ with $N=0,1,2,3$, or 4 for each molecule. The shifts introduced are of the order of a few kilohertz. The effect is only a shift in frequency but the general structure of the schemes is not affected. Intermolecular and intramolecular gates could still be driven with similar pulses in a larger network.

The hyperfine structure of rovibrational molecules can complicate the manipulation of molecules with microwave pulses because unwanted transitions can occur [52,53]. In the present model, the hyperfine structure was not taken into account. Within the rotational level $N=0$ manifold, the most important term is the scalar hyperfine coupling proportional to $\mathbf{i}_{1} \cdot \mathbf{i}_{2}$, where $\mathbf{i}_{1}$ and $\mathbf{i}_{2}$ are the nuclear spins of the atoms of the molecule. The splitting between hyperfine levels is typically of the order of a kilohertz. For $N$ different than 0 , the most important contribution to the hyperfine Hamiltonian comes from the nuclear quadrupolar interaction which gives a splitting of the order of a few hundreds of kilohertz. In our case, $i_{\mathrm{Na}}=3 / 2$, and $i_{\mathrm{Cs}}=7 / 2 . N=0$ splits into 32 states, and $N=1$ splits into 96 levels. For a static electric field of $2 \mathrm{kV} / \mathrm{cm}$, more than four rotational levels per vibrational manifold are needed to described accurately the energy structure. Taking into account all the hyperfine levels would complicate the theoretical simulations since we had to manipulate the tensor product of the individual basis. However, the hyperfine structure won't change the conclusion of this work. Simulations of the logical operations showed two different characteristic time scales for the logic gates. Fast gates can be driven with pulses no longer than a few tens of nanoseconds. These pulses are broad enough that all hyperfine transitions within one rotational level will be excited equally, and the unresolved hyperfine structure can be neglected. On the other hand, slower gates such as the TOFFOLI of the adder algorithm are driven by much narrower pulses, and the splitting between hyperfine levels, larger than the dipole-dipole interaction splitting, will be well resolved and only one hyperfine level will be active. We note as well that as the static electric field 
increases orientating the molecules along the electric field, $m_{N}$ becomes a fairly good quantum number, and the matrix of the transition dipole moments between hyperfine levels of different rotational manifolds shows that, for an electric field typically stronger than $1 \mathrm{kV} / \mathrm{cm}$, only a few transitions are allowed, reducing considerably the possibilities for unwanted transitions.

Other terms that we have not considered are the secondorder Stark effects resulting from the static electric and the optical trapping potential. The static second-order Stark effect is of the order of a few kilohertz for the diagonal terms. The effect due to the laser field can be more important due to the wavelength dependence of the molecular polarizabilities. For a laser intensity typically of the order of $1 \mathrm{~kW} / \mathrm{cm}^{2}$, and a molecular polarizabilty of a few thousands of atomic units, the second-order Stark effect is of the order of $100 \mathrm{kHz}$ for the diagonal terms. It is not strong enough to affect our results.

The numerical simulations suggest that ultracold trapped polar molecules are promising for the concatenation of several gates with a high fidelity. First, we can compare the efficiency of a couple of diatomic systems with a tetra-atomic molecule to implement arithmetic operations. It was not easy to encode the four qubits of the full adder in the two interacting dimers. Some hundreds of microseconds to add 1 is finally much longer than the time scale of the full addition in a polyatomic entity (some tens of ps). However the main point is the possibility to avoid intermediary reading out and re-encoding of the carry out. Here the system is directly ready for the next cycle involving the next molecule. This is a step toward scalability but at the price of long pulse duration. The other advantage is the simplicity and robustness of the scheme based on $\pi$ pulses.

\section{PHASE-CORRECT GATES}

The relative phase of the gate transitions optimized among the states of the computational basis set can reduce the accuracy of the gate when it is applied to an arbitrary superposed state. $\pi$ pulses are not sufficient to give phase-correct quantum gates. Then correct gate pulses can be determined by the optimal control theory generalized to the multitarget case [7] with a phase constraint $[19,54]$.

\section{A. Multitarget optimal control}

The optimal field maximizes the objective functional $J$ with the constraints that the Schrödinger equation is satisfied at any time $[55,56]$ and that the laser pulse intensity is not too high. The functional reads

$$
\begin{aligned}
J= & \sum_{n=1}^{Z}\left|\left\langle\psi_{i}^{n}\left(t_{f}\right) \mid \phi_{f}^{n}\right\rangle\right|^{2}-2 \operatorname{Re}\left[\int_{0}^{t}\left\langle\psi_{f}^{n}(t)\left|\partial_{t}+\frac{i}{\hbar} H\right| \psi_{i}^{n}(t)\right\rangle\right] \\
& -\alpha \int_{0}^{t_{f}} E^{2}(t) d t
\end{aligned}
$$

where $\alpha$ is a positive penalty factor chosen to weight the importance of the laser fluence. For an $N$-qubit gate, $Z=$ $2^{N}+1$, where $2^{N}$ is the number of input-output transitions in the gate transformations and the supplementary equation is the phase constraint. The $\psi_{i}^{n}(t)$ are the wave packets which are propagated forward in time with the initial conditions $\psi_{i}^{n}(t=0)=\phi_{i}^{n}, n=1, \ldots, Z$. The Lagrange multipliers $\psi_{f}^{n}(t)$ are propagated backward in time with the final conditions $\psi_{f}^{n}\left(t=t_{f}\right)=\phi_{f}^{n}, n=1, \ldots, Z$. The supplementary transfer which imposes the phase correction is a sum over all the transitions of the gate [19]:

$$
\frac{1}{\sqrt{2^{N}}} \sum_{k=1}^{2^{N}}\left|\psi_{i}^{k}\right\rangle \rightarrow \frac{1}{\sqrt{2^{N}}}\left[\sum_{j=1}^{2^{N}}\left|\psi_{f}^{k}\right\rangle\right] e^{i \phi},
$$

and the single phase $\phi$ can take any value between 0 and $2 \pi$. The universal gate field is a sum of $Z$ contributions:

$$
E_{j}(t)=-(s(t) / \hbar \alpha) \operatorname{Im}\left[\sum_{n=1}^{Z}\left\langle\psi_{f}^{n}(t)\left|\mu_{j}\right| \psi_{i}^{n}(t)\right\rangle\right],
$$

where $j$ denotes the polarization direction of the electric field. The fidelity well adapted to take into account the phases is given by

$$
F=\frac{1}{Z^{2}}\left|\sum_{n}^{Z}\left\langle\psi_{i}^{n}(t) \mid \phi_{f}^{n}\right\rangle\right|^{2}
$$

\section{B. Deutsch-Jozsa algorithm}

The Deutsch-Jozsa algorithm for a one-qubit function will serve us to illustrate the realization of phase-correct gates.

The Deutsch-Jozsa's problem illustrates the speedup of quantum computing by taking advantage of superposed states. It has been the subject of several theoretical studies $[10,12,17,19,24,30]$ and has been experimentally implemented [8,57-59]. The principles can be summarized as follows: we suppose that a function applied on one qubit $|x\rangle$ can either change its value (balanced function) or leave it unchanged (constant function). The problem is to determine whether the function is balanced or constant by a single call to the function and one measure. The logical circuit for the Deutsch-Jozsa algorithm is sketched in Fig. 10. The algorithm requires an auxiliary qubit $|y\rangle$. After a NOT gate on $|y\rangle$, the two qubits are put in a superposed state by one Hadamard gate on each qubit. The Haddamard gate superposes the qubit states according to

$$
\begin{aligned}
& |0\rangle \stackrel{\mathrm{HAD}}{\longrightarrow}(1 / \sqrt{2})(|0\rangle+|1\rangle), \\
& |1\rangle \stackrel{\mathrm{HAD}}{\longrightarrow}(1 / \sqrt{2})(|0\rangle-|1\rangle) .
\end{aligned}
$$

The call function is then implemented by applying the transformation $U_{f}$ :

$$
U_{f}:|x\rangle|y\rangle \rightarrow|x\rangle|y \oplus f(x)\rangle .
$$

A final Hadamard gate is carried out on $|x\rangle$. When the initial state is $|00\rangle$, the final state of $|x\rangle$ is $\pm|0\rangle$ for a constant function and $\pm|1\rangle$ for a balanced function. The nature of the function is therefore determined by only one query.

We simulate the example with the balanced function $f(0)=1$ and $f(1)=0$. In this case $U_{f}$ is the CNOT gate with control qubit $|x\rangle$. Qubits $|x\rangle$ and $|y\rangle$ are encoded in the first two rotational states of two neighboring molecules in the vibrational state $v=0:|x\rangle|y\rangle \leftrightarrow\left|\tilde{N}_{1} \tilde{N}_{2}\right\rangle$, with $v_{1}=v_{2}=0$ (see Fig. 2). The basis set for the simulation contains the vibrational state $v=0$ and rotational states up to $N=4$ for 


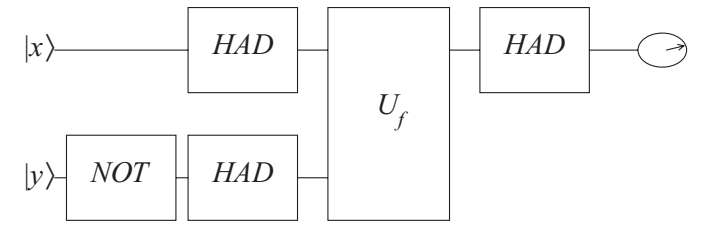

FIG. 10. Logical circuit for the Deutsch-Jozsa algorithm.

each molecule. Both Hadamard steps involve intramolecular transitions and the frequencies are mainly fixed by the Stark levels so that the pulse duration is shorter than that for the intermolecular CNOT gate which is the bottleneck of the full algorithm.

\section{NOT-HADHAD step}

Optimal control allows us to optimize a global gate driving the resulting transformation of the first NOT gate on $|y\rangle$ and the two HAD gates on both qubits $|x\rangle$ and $|y\rangle$ in a single shot as suggested in previous works [25-28]. This provides an interesting speedup. This NOT-HADHAD transformation in the coupled basis set is

$$
\begin{aligned}
& \left|\tilde{0} \tilde{0}>\rightarrow 2^{-1}(|\tilde{0} \tilde{0}\rangle-|\tilde{0} \tilde{1}\rangle+|\tilde{1} \tilde{0}\rangle-|\tilde{1} \tilde{1}\rangle),\right. \\
& \left|\tilde{0} \tilde{1}>\rightarrow 2^{-1}(|\tilde{0} \tilde{0}\rangle+|\tilde{0} \tilde{1}\rangle+|\tilde{1} \tilde{0}\rangle+|\tilde{1} \tilde{1}\rangle),\right. \\
& \left|\tilde{1} \tilde{0}>\rightarrow 2^{-1}(|\tilde{0} \tilde{0}\rangle+|\tilde{0} \tilde{1}\rangle-|\tilde{1} \tilde{0}\rangle-|\tilde{1} \tilde{1}\rangle),\right. \\
& \left|\tilde{1} \tilde{1}>\rightarrow 2^{-1}(|\tilde{0} \tilde{0}\rangle-|\tilde{0} \tilde{1}\rangle-|\tilde{1} \tilde{0}\rangle+|\tilde{1} \tilde{1}\rangle),\right.
\end{aligned}
$$

and the additional phase equation is

$$
\left.2^{-1}(|\tilde{0} \tilde{0}\rangle+|\tilde{0} \tilde{1}\rangle)+|\tilde{1} \tilde{0}\rangle+|\tilde{1} \tilde{1}\rangle\right) \rightarrow|\tilde{0} \tilde{0}\rangle .
$$

The NOT-HADHAD gate has been implemented by optimal control without guess field and a pulse duration of $\tau_{p}=63 \mathrm{~ns}$. A fidelity of 0.99999 is reached after 400 iterations. The phases are optimized within $3 \times 10^{-3} \pi$. The evolution of the populations starting from a superposed state with equal weights 0.25 on the computational basis set states $|\tilde{0} \tilde{0}\rangle,|\tilde{0} \tilde{1}\rangle$, $|\tilde{1} \tilde{0}\rangle$, and $|\tilde{1} \tilde{1}\rangle$ is shown in the top panel of Fig. 11. The corresponding optimal field and its Fourier transform are given in Fig. 12.

\section{Phase-correct intermolecular CNOT gate}

The supplementary transformation to ensure that the phases of the final states of each transition are equal is

$$
\begin{aligned}
& \frac{1}{2}(|00\rangle+|01\rangle+|10\rangle+|11\rangle) \\
& \quad \rightarrow \frac{1}{2}\left(|00\rangle e^{i \varphi_{1}}+|01\rangle e^{i \varphi_{2}}+|10\rangle e^{i \varphi_{3}}+|11\rangle e^{i \varphi_{4}}\right),
\end{aligned}
$$

with

$$
\varphi_{1}=\varphi_{2}=\varphi_{3}=\varphi_{4}=\varphi
$$

where the phase $\varphi$ can take any value between 0 and $2 \pi$. The $\pi$ pulse is a very good trial field since the convergence is fast with a fidelity index of 0.99943 in 130 iterations. Figure 13 compares the population evolution starting from a superposed state driven by the $\pi$ pulse (see Fig. 3) or by the optimal control field. The fidelity for the transformations of the states $|\tilde{0} \tilde{0}\rangle$ and $|\tilde{0} \tilde{1}\rangle$ decreases with the $\pi$ pulse. Owing to the phase constraint, the fidelity is better with optimal control.

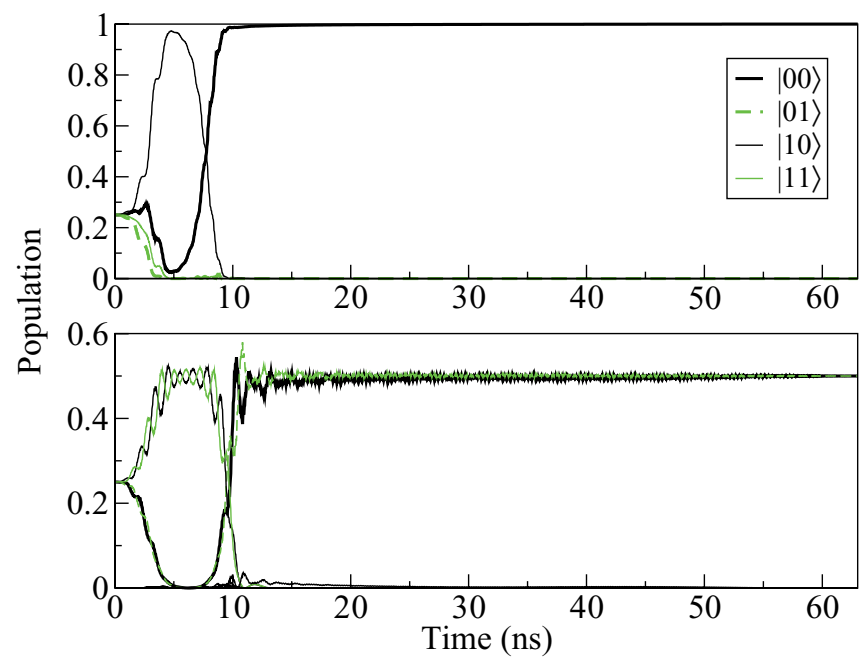

FIG. 11. (Color online) Evolution of the populations during the gates involving Hadamard gates in the Deutsch-Jozsa algorithm. Top panel: NOT-HADHAD step (NOT gate on $|y\rangle$ and the two HAD gates on $|x\rangle$ and $|y\rangle$. Bottom panel: HAD gate on $|x\rangle$. The initial state is a superposed state with equal weights 0.25 on the computational basis set states $|\tilde{0} \tilde{0}\rangle$ (thick solid black curve), $|\tilde{0} \tilde{1}\rangle$ (thick solid green curve), $|\tilde{1} \tilde{0}\rangle$ (thin solid black curve), $|\tilde{1} \tilde{1}\rangle$ (thin solid green curve).

The phases obtained for the fifth transformation (41) are $\varphi_{1}=0.202 \pi, \varphi_{2}=0.199 \pi, \varphi_{3}=0.200 \pi$, and $\varphi_{4}=0.202 \pi$. The optimal control has found field amplitudes of the same order of magnitude as for the $\pi$ pulse. The main frequency is the carrier frequency of the $\pi$ pulse and a lot of very small frequencies without direct signification from the model.

\section{E. Hadamard gate on $|x\rangle$}

One has to consider the extended transformation in a twoqubit space here. The HAD gate (last step) on $|x\rangle$ becomes in the coupled basis set:

$$
|\tilde{0} \tilde{0}\rangle \rightarrow 2^{-1 / 2}(|\tilde{0} \tilde{0}\rangle+|\tilde{0} \tilde{1}\rangle)
$$
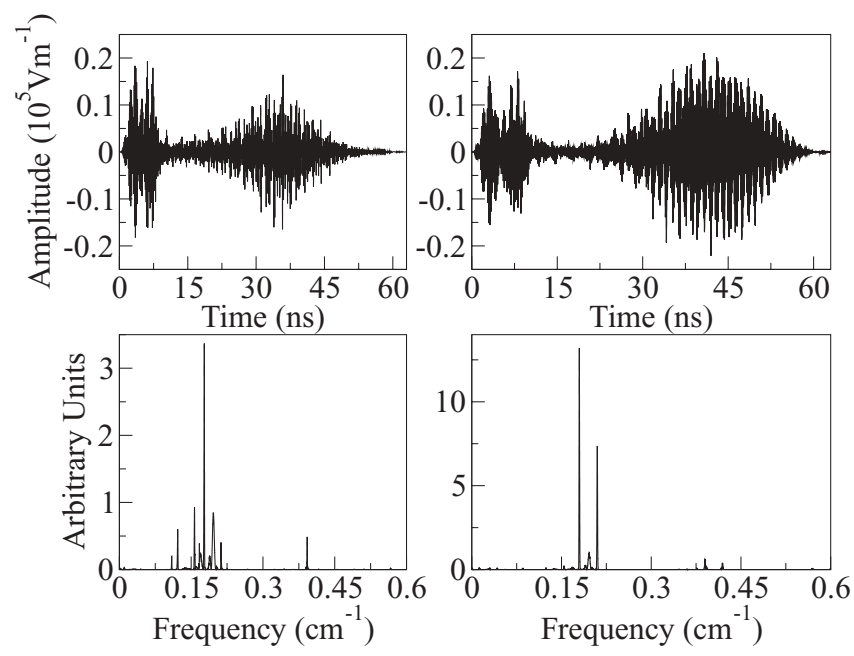

FIG. 12. Amplitude of the optimal field for the NOT-HADHAD gate (top left panel) and for the HAD gate (top right panel). Bottom panel: The corresponding Fourier transforms. 


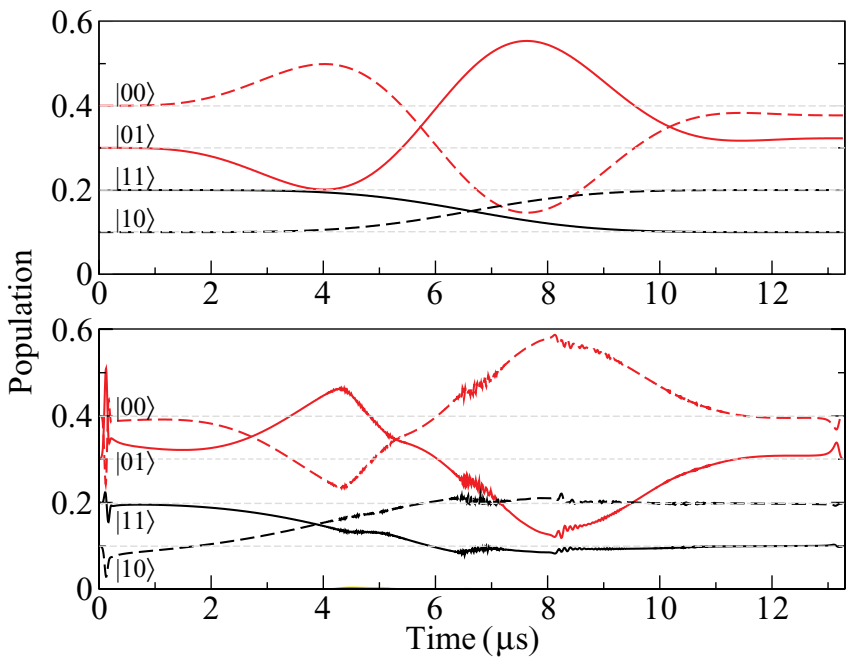

FIG. 13. (Color online) Evolution of the populations during the phase CNOT gate starting from a superposition of states $|\tilde{0} \tilde{0}\rangle$ (red dashed curve), $|\tilde{0} \tilde{1}\rangle$ (solid red curve), $|\tilde{1} \tilde{0}\rangle$ (black dashed curve), and $|\tilde{1} \tilde{1}\rangle$ (black solid curve). Top panel: Gate driven by the $\pi$ pulse calculated in Fig. 3. The pulse optimized for a pure state does not lead to a high-fidelity operation when applied to a superposition of states. Bottom panel: High-fidelity gate optimized by optimal control. We checked that high-fidelity gates were obtained when the pulses were applied to other initial superpositions.

$$
\begin{aligned}
& |\tilde{0} \tilde{1}\rangle \rightarrow 2^{-1 / 2}(|\tilde{0} \tilde{1}\rangle+|\tilde{1} \tilde{1}\rangle), \\
& |\tilde{1} \tilde{0}\rangle \rightarrow 2^{-1 / 2}(|\tilde{0} \tilde{0}\rangle-|\tilde{1} \tilde{0}\rangle), \\
& |\tilde{1} \tilde{1}\rangle \rightarrow 2^{-1 / 2}(|\tilde{0} \tilde{1}\rangle-|\tilde{1} \tilde{1}\rangle),
\end{aligned}
$$

with the additional equation for the phase constraint,

$$
(|\tilde{0} \tilde{0}\rangle+|\tilde{0} \tilde{1}\rangle+|\tilde{1} \tilde{0}\rangle+|\tilde{1} \tilde{1}\rangle) \rightarrow 2^{-1 / 2}(|\tilde{0} \tilde{0}\rangle+|\tilde{0} \tilde{1}\rangle) .
$$

The evolution of the populations starting from a superposed state with equal weights 0.25 on the computational basis set states $|\tilde{0} \tilde{0}\rangle,|\tilde{0} \tilde{1}\rangle,|\tilde{1} \tilde{0}\rangle$, and $|\tilde{1} \tilde{1}\rangle$ is shown in the bottom panel
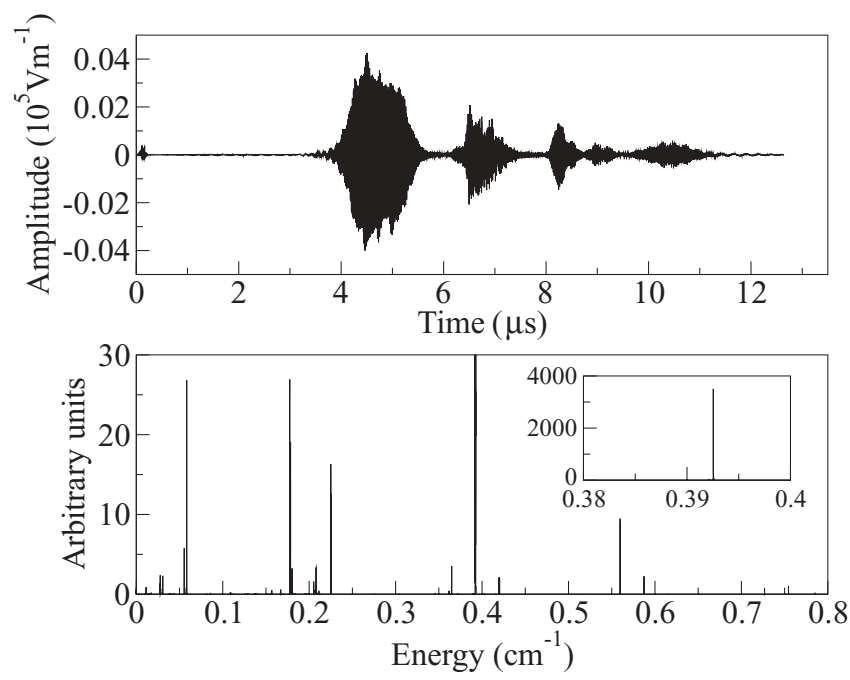

FIG. 14. Amplitude of the optimal field for the CNOT gate (top panel) and its Fourier transform (bottom panel). of Fig. 11. The corresponding optimal field and its Fourier transform are given in Fig. 12. As before, a fidelity of 0.99998 is obtained after 400 iterations. The phases are optimized within $10^{-2} \pi$.

Final optimal fields driving the desired operations can have a complicated envelop and differ drastically from the initial $\pi$ pulse, raising the question of the robustness and actual realization of such pulses. For schemes involving only rotational levels, the Fourier transform of the pulses shows that the spectrum contains frequencies in the microwave region only. In the case of the Hadamard gates (see Fig. 12), frequencies are within $0.1 \mathrm{~cm}^{-1}(3 \mathrm{GHz})$ and $0.2 \mathrm{~cm}^{-1}$ $(6 \mathrm{GHz})$. For this range of frequencies, electronic equipment can generate any arbitrary pulses and therefore obtaining such optimized pulses is experimentally feasible. Because several rotational levels are taken into account in the model (up to $N=4$ in the present study), optimized pulses may contain frequencies that will be more challenging to obtain. In the case of the optimized CNOT gate (see Fig. 14), the pulse contains frequencies up to $0.75 \mathrm{~cm}^{-1}(20 \mathrm{GHz})$. Filters can be used during the optimization procedure to eliminate out-of-range frequencies. If different vibrational levels are used. Pulses will be in the infrared region where pulse shaping techniques are less developed.

\section{CONCLUSION}

Manipulating trapped ultracold molecules by laser fields offers great potential toward scalable quantum computing. Logical operations can be performed by splitting processes into intramolecular global gates and intermolecular gates. The latter are very crucial for scalable operations for they enable the transfer of information from one molecule to another through the dipole-dipole interaction. Using the states of the coupled basis set as logical states complicates the realization of onequbit or two-qubit gates when the number of qubits involved in the logical operations increases. This can be overcome by using schemes that involve switchable interactions. Storing molecules in rovibrational levels with vanishing average dipole moment and transferring them when needed to levels with strong dipole moments by adiabatic passage techniques could be a possibility. This operation would not take any longer than a few microseconds and would be equivalent in terms of duration to an additional intermolecular gate. The implementation of schemes that would include switchable interactions would further extend the potential of polar molecules for scalable quantum information. This remains open and can stimulate new research in molecular design, storage, and communication among entities.

\section{ACKNOWLEDGMENTS}

The authors wish to thank J.M. Teuler for technical assistance. This research was supported by Triangle de la Physique under Contract 20009-038T. The computing facilities with the financial support of the FRS for the University of Liège "Nic3" project are also acknowledged. 
[1] L. Isenhower, E. Urban, X. L. Zhang, A. T. Gill, T. Henage, T. A. Johnson, T. G. Walker, and M. Saffman, Phys. Rev. Lett. 104, 010503 (2010).

[2] T. Monz, K. Kim, W. Hansel, M. Riebe, A. S. Villar, P. Schindler, M. Chwalla, M. Hennrich, and R. Blatt, Phys. Rev. Lett. 102, 040501 (2009).

[3] L. DiCarlo et al., Nature (London) 460, 240 (2009).

[4] A. Politi, J. C. F. Matthews, and J. L. O’Brien, Science 325, 1221 (2009).

[5] J. Du, N. Xu, X. Peng, P. Wang, S. Wu, and D. Lu, Phys. Rev. Lett. 104, 030502 (2010).

[6] C. M. Tesch, L. Kurtz, and R. de Vivie-Riedle, Chem. Phys. Lett. 343, 633 (2001).

[7] C. M. Tesch and R. de Vivie-Riedle, Phys. Rev. Lett. 89, 157901 (2002).

[8] J. Vala, Z. Amitay, B. Zhang, S. R. Leone, and R. Kosloff, Phys. Rev. A 66, 062316 (2002).

[9] D. Babikov, J. Chem. Phys. 121, 7577 (2004).

[10] Y. Ohtsuki, Chem. Phys. Lett. 404, 126 (2005).

[11] C. Menzel-Jones and M. Shapiro, Phys. Rev. A 75, 052308 (2007).

[12] K. Shioya, K. Mishima, and K. Yamashita, Mol. Phys. 105, 1287 (2007).

[13] K. Mishima, K. Tokumo, and K. Yamashita, Chem. Phys. 343, 61 (2008).

[14] M. Tsubouchi and T. Momose, Phys. Rev. A 77, 052326 (2008).

[15] M. Tsubouchi, A. Khramov, and T. Momose, Phys. Rev. A 77, 023405 (2008).

[16] D. Sugny, L. Bomble, T. Ribeyre, O. Dulieu, and M. DesouterLecomte, Phys. Rev. A 80, 042325 (2009).

[17] K. Mishima and K. Yamashita, Chem. Phys. 367, 63 (2010).

[18] R. Zaari and A. Brown, J. Chem. Phys. 132, 014307 (2010).

[19] C. M. Tesch and R. de Vivie-Riedle, J. Chem. Phys. 121, 12158 (2004).

[20] U. Troppmann and R. de Vivie-Riedle, J. Chem. Phys. 122, 154105 (2005).

[21] B. Korff, U. Troppmann, K. Kompa, and R. de Vivie-Riedle, J. Chem. Phys. 123, 244509 (2005)

[22] D. Sugny, C. Kontz, M. Ndong, Y. Justum, G. Dive, and M. Desouter-Lecomte, Phys. Rev. A 74, 043419 (2006).

[23] D. Sugny, M. Ndong, D. Lauvergnat, Y. Justum, and M. Desouter-Lecomte, J. Photochem. Photobiol. A 190, 359 (2007).

[24] M. Ndong, D. Lauvergnat, X. Chapuisat, and M. DesouterLecomte, J. Chem. Phys. 126, 244505 (2007).

[25] D. Weidinger and M. Gruebele, Mol. Phys. 105, 1999 (2007).

[26] L. Bomble, D. Lauvergnat, F. Remacle, and M. DesouterLecomte, J. Chem. Phys. 128, 064110 (2008).

[27] L. Bomble, D. Lauvergnat, F. Remacle, and M. DesouterLecomte, Phys. Rev. A 80, 022332 (2009).

[28] L. Bomble, D. Lauvergnat, F. Remacle, and M. DesouterLecomte, Phys. Chem. Chem. Phys. 12, 15628 (2010).

[29] M. Schröder and A. Brown, J. Chem. Phys. 131, 034101 (2009).

[30] K. Mishima and K. Yamashita, Chem. Phys. 361, 106 (2009).

[31] K. Mishima and K. Yamashita, J. Chem. Phys. 130, 034108 (2009).
[32] D. DeMille, Phys. Rev. Lett. 88, 067901 (2002).

[33] L. D. Carr, D. DeMille, R. V. Krems, and J. Ye, New J. Phys. 11, 055049 (2009).

[34] S. F. Yelin, K. Kirby, and R. Côté, Phys. Rev. A 74, 050301(R) (2006).

[35] E. Kuznetsova, R. Côté, K. Kirby, and S. F. Yelin, Phys. Rev. A 78, 012313 (2008).

[36] E. Charron, P. Milman, A. Keller, and O. Atabek, Phys. Rev. A 75, 033414 (2007).

[37] D. Beckman, A. N. Chari, S. Devabhaktuni, and J. Preskill, Phys. Rev. A 54, 1034 (1996).

[38] D. Deutsch and R. Jozsa, Proc. R. Soc. London A 439, 553 (1992).

[39] M. Aymar and O. Dulieu, J. Chem. Phys. 122, 204302 (2005).

[40] C. Haimberger, J. Kleinert, M. Bhattacharya, and N. P. Bigelow, Phys. Rev. A 70, 021402 (2004).

[41] M. Korek, A. R. Allouche, K. Fakhreddine, and A. Chaalan, Can. J. Phys. 78, 977 (2000).

[42] S. Kotochigova and E. Tiesinga, Phys. Rev. A 73, 041405(R) (2006).

[43] A. Micheli, G. Pupillo, H. P. Büchler, and P. Zoller, Phys. Rev. A 76, 043604 (2007).

[44] C. Cohen-Tannoudji, B. Diu, and F. Laloë, Mécanique Quantique (Hermann, Paris, 1973).

[45] W. H. Press, S. A. Teukolsky, W. T. Vetterling, and B. P. Flannery, Numerical Recipes in FORTRAN, The Art of Scientific Computing, 2nd ed. (Cambridge University Press, Cambridge, UK, 1986).

[46] A. Barenco, D. Deutsch, A. Ekert, and R. Jozsa, Phys. Rev. Lett. 74, 4083 (1995).

[47] G. P. Berman, D. K. Campbell, G. D. Doolen, G. V. López, and V. I. Tsifrinovich, Physica B 240, 61 (1997).

[48] N. Rosen and C. Zener, Phys. Rev. 40, 502 (1932).

[49] G. S. Vasilev and N. V. Vitanov, Phys. Rev. A 70, 053407 (2004).

[50] V. Vedral, A. Barenco, and A. Ekert, Phys. Rev. A 54, 147 (1996).

[51] G. Benenti, G. Casati, and G. Strini, Principles of Quantum Computation and Information (World Scientific, Singapore, 2004).

[52] J. Aldegunde, H. Ran, and J. M. Hutson, Phys. Rev. A 80, 043410 (2009).

[53] H. Ran, J. Aldegunde, and J. M. Hutson, New J. Phys. 12, 043015 (2010).

[54] M. Zhao and D. Babikov, J. Chem. Phys. 125, 024105 (2006).

[55] W. Zhu and H. Rabitz, J. Chem. Phys. 109, 385 (1998).

[56] Y. Ohtsuki, Y. Teranishi, P. Saalfrank, G. Turinici, and H. Rabitz, Phys. Rev. A 75, 033407 (2007).

[57] S. Takeuchi, Phys. Rev. A 62, 032301 (2000).

[58] K. Dorai, Arvind, and A. Kumar, Phys. Rev. A 61, 042306 (2000).

[59] C. Ju, J. Zhu, X. Peng, B. Chong, X. Zhou, and J. Du, Phys. Rev. A 81, 012322 (2010). 